\title{
Association of low plasma antioxidant levels with all-cause mortality and coronary events in healthy middle-aged men from France and Northern Ireland in the PRIME study
}

\author{
Gareth J. McKay ${ }^{1}{ }^{10} \cdot$ Natalie Lyner $^{1} \cdot$ Gerry J. Linden ${ }^{1} \cdot$ Frank Kee $^{1} \cdot$ Marie Moitry $^{2} \cdot$ Katia Biasch $^{2} \cdot$ \\ Philippe Amouyel $^{3}$. Jean Dallongeville ${ }^{3} \cdot$ Vanina Bongard $^{4} \cdot$ Jean Ferrières ${ }^{4} \cdot$ K. Fred Gey $^{5} \cdot$ Chris C. Patterson $^{1}$. \\ Jayne V. Woodside ${ }^{1}$
}

Received: 24 July 2020 / Accepted: 3 December 2020 / Published online: 23 December 2020

(c) The Author(s) 2020

\begin{abstract}
Background The main underlying risk factors associated with coronary heart disease (CHD) are modifiable and oxidative injury and systemic inflammatory damage represent key aetiological factors associated with the development and progression of CHD and premature mortality.

Objective To examine associations of plasma antioxidant status with all-cause mortality and fatal or non-fatal cardiovascular events.

Design The PRIME study prospectively evaluated 9709 men aged 50-59 years between 1991 and 1993 in Northern Ireland and France who were free of CHD at recruitment and followed annually for deaths and cardiovascular events for 10 years. Serum concentrations of vitamin C, retinol, two forms of vitamin E ( $\alpha$ - and $\gamma$-tocopherol) and six carotenoids were quantified by high-performance liquid chromatography. Baseline conventional risk factors were considered, as well as socioeconomic differences and lifestyle behaviours including diet, smoking habit, physical activity, and alcohol consumption through Cox regression analyses.

Results At 10 years, there were 538 deaths from any cause and 440 fatal or non-fatal cardiovascular events. After adjustment for country, age, systolic blood pressure, diabetes, body mass index, cholesterol, high density lipoprotein cholesterol, triglycerides, height, total physical activity, alcohol consumption and smoking habit, higher levels of all antioxidants were associated with significantly lower risk of all-cause mortality, with the exception of $\gamma$-tocopherol. Only retinol was significantly associated with decreased risk of cardiovascular events in a fully adjusted model.

Conclusions Low antioxidant levels contribute to the gradient of all-cause mortality and cardiovascular incidence independent of lifestyle behaviours and traditional cardiovascular and socioeconomic risk factors.
\end{abstract}

Keywords Cardiovascular disease $\cdot$ Premature mortality $\cdot$ Antioxidant $\cdot$ Vitamin $\cdot$ Carotenoid $\cdot$ Retinol

Supplementary Information The online version contains supplementary material available at https://doi.org/10.1007/s0039 4-020-02455-2.

Gareth J. McKay

g.j.mckay@qub.ac.uk

1 Centre for Public Health, Royal Victoria Hospital, Queen's University Belfast, Belfast BT12 6BA, UK

2 MONICA-Strasbourg, Strasbourg, France

3 MONICA-Lille, Lille, France

4 MONICA-Toulouse, Toulouse, France

5 The MONICA Reference Centre for Vitamins, Department of Biochemistry and Molecular Biology, University of Bern, Bern, Switzerland

\section{Introduction}

Cardiovascular disease (CVD) is the leading global cause of death accounting for an estimated 17.9 million deaths in 2016 according to the World Health Organisation. This represents $31 \%$ of all deaths worldwide, a figure expected to increase with rising obesity and diabetes prevalence, leading to a greater burden on healthcare provision [1]. The main underlying risk factors associated with CVD are modifiable and evidence has implicated oxidative injury and systemic inflammatory damage as key aetiological factors in both the development and progression of CVD pathogenesis [2-4]. A high intake of fruit, vegetables and 
nuts has been associated with a reduction in CVD risk and premature mortality [5-7], with nutrition and diet estimated to account for approximately $45 \%$ of all adult cardiometabolic deaths in the United States in 2012 [8]. Biomarker measurement provides improvements over estimates of nutritional intake, strengthening the reliability of association studies in nutritional epidemiology [9]. Nevertheless, the influence of dietary antioxidants on CVD risk and all-cause mortality remains unclear [10]. LDLcholesterol becomes more atherogenic following oxidative modification, leading to its accumulation on arterial walls, while antioxidants have been shown to reduce the rate of progression of atherosclerosis [11, 12]. Fat-soluble antioxidants such as vitamins $\mathrm{A}$ and $\mathrm{E}$, as well as the serum carotenoids, are carried in the fatty core of LDL particles, prolonging LDL resistance to oxidative damage [12]. Plausible mechanisms by which nutritional factors, such as serum carotenoids and vitamins, may reduce CVD risk and premature mortality, have been proposed, comprising antioxidative and/or anti-inflammatory processes that counteract the effects of free radicals, including those common to vascular and non-vascular diseases [13]. Nevertheless, randomised supplementation trials that are largely limited to single antioxidant evaluation, have failed to consistently report reduced risk associated with CVD events and all-cause mortality identified through observational epidemiological studies, with some reporting unanticipated harmful effects [14]. While reduced all-cause mortality has been reported in nutritionally deficient individuals with low plasma antioxidant levels following supplementation, excess levels in sufficient individuals may not offer additional benefit and possibly result in potentially toxic effects [15-17].

Contrasting lifestyle and dietary behaviours, including antioxidant bioavailability, may contribute to variation in CVD risk and all-cause mortality as previously reported for the higher incidence of ischaemic heart disease in Northern Ireland (NI) compared to France [18, 19]. We hypothesised that variation in plasma retinol, alpha- and gamma-tocopherol (referred to as antioxidant vitamins), vitamin $\mathrm{C}$, as well as the six major serum-based carotenoids: lutein, zeaxanthin, beta-cryptoxanthin, lycopene, alpha- and beta-carotene may be associated with cardiovascular and mortality outcomes in participants from the Prospective Epidemiological Study of Myocardial Infarction (PRIME). The PRIME study prospectively examined middle-aged men in France and NI, countries with contrasting culture and lifestyle. The aim of this study was to evaluate associations between levels of exogenous antioxidants in the plasma of study participants characterised as free from CVD at the point of recruitment with the incidence of coronary events and all-cause mortality over a 10-year follow-up period.

\section{Methods}

\section{Participants}

The PRIME Study is a multi-centre, prospective cohort composed of men aged 50-59 years when recruited from the general population of Belfast in NI and Lille, Strasbourg and Toulouse in France, between 1991 and 1993 $[20,21]$. A detailed medical, lifestyle and food frequency questionnaire (FFQ) was completed at baseline examination which included information on lifetime smoking, physical activity [22], alcohol consumption [23] and diet [24]. A cardiovascular screening examination was also conducted at baseline, which included a detailed history of previous CVD. Follow-up included annual questionnaires for 10 years and possible cardiovascular events were confirmed by a medical committee [21, 25]. Of the 10,600 men recruited, 317 (3.0\%) were lost to follow-up after 10 years, 215 (2\%) withdrew their consent for participation within the study, and $653(6.2 \%)$ died during this time. Cardiovascular events included validated myocardial infarction and/or stroke (fatal and non-fatal). Death certificates were obtained for all men who died and causes of death were categorised using the International Classification of Diseases (ICD) Ninth Revision.

\section{Serum antioxidant measurement}

Fasting venous blood samples were drawn at baseline and centrifuged within $4 \mathrm{~h}$ of collection. Plasma samples were aliquoted for long-term storage at $-80{ }^{\circ} \mathrm{C}$. Plasma concentrations of retinol, $\alpha$-tocopherol, $\gamma$-tocopherol, and six carotenoids ( $\alpha$-carotene, $\beta$-carotene, $\beta$-cryptoxanthin, lutein, lycopene, zeaxanthin) were determined by high performance liquid chromatography (HPLC) with diode array detection [26]. Results were analysed using Chromquest 4.2 software (Thermo Fisher Scientific, Massachusetts, USA). Standards used in the assay were supplied by either Sigma (Sigma-Aldrich, Poole, Dorset, UK) or Chemos (Chemos GmbH, Regensburg, Germany). Vitamin $\mathrm{C}$ was measured in plasma which had been stabilised on collection on a BMG FLUOstar Optima plate reader adapted from the method by Vuilleumier and Keck [27]. These assays were validated against the National Institute of Standards and Technology (NIST) Standard Reference Material 968d for fat-soluble vitamins, carotenoids and cholesterol and NIST Standard Reference Material 970 for ascorbic acid in human serum, which were externally quality assured three times per year using samples supplied by the French Society for Vitamins and Biofactors. In-house quality control samples were also included in every run. 
The inter- and intra-assay coefficients of variation (CV) for vitamin $\mathrm{A}, \mathrm{E}$ and carotenoid assays were $<15 \%$ and $<7.5 \%$ for vitamin C. A mean standardised antioxidant score (SAS) was estimated from the mean standardised $Z$ scores for the ten logarithmically transformed antioxidants.

\section{Lifestyle variables}

Body mass index (BMI) was calculated as the body weight/ height $^{2}\left(\mathrm{~kg} / \mathrm{m}^{2}\right)$. Lifetime smoking was expressed in five categories: never smoked, smoked other than cigarettes, smoked $<15$ cigarette pack years, smoked $\geq 15$ but $<30$ cigarette pack years and smoked $\geq 30$ cigarette pack years. Alcohol intake was also expressed as five categories of consumption; none, 1-128, 129-265, 266-461 and $\geq 462 \mathrm{~mL} /$ week. Physical activity (in metabolic equivalent of task (METs) scores/week) was positively skewed and was therefore summarised using median and interquartile range. Diabetes was defined by self-report. Socioeconomic status was categorised into high, middle and low using a composite measure of material conditions based on the type of living accommodation (rented or owned, mortgaged), number of cars/vans/motorcycles in the household and the number of baths and/or showers and toilets in the home [22].

The daily fruit, vegetable and juice (FVJ) score included all fruit and vegetable variables from the PRIME FFQ. This involved eight categories: 'salad', 'carrot', 'tomato', 'natural' and 'baked vegetables', 'citrus fruits', 'other fruits' and a 'juice' variable. Potatoes were not included as they were included under the 'Bread, cereals and potatoes' food group. Frequencies of each variable were transformed into daily consumption values (the 'juice' variable was limited to a maximum of one daily portion in-line with UK dietary guidelines) and these new variables were summed for each participant to give total daily FVJ consumption. This variable was positively skewed and was therefore summarised using median and interquartile range.

Ethical approval for baseline examination and follow-up was obtained at each study centre; all participants provided informed consent and the study was compliant with the Declaration of Helsinki guidelines.

\section{Statistical methods}

Data for antioxidant levels were not normally distributed and were therefore logarithmically transformed before analysis. Results were summarised using the geometric mean and inter-quartile range. Correlation coefficients between fruit and vegetable consumption and antioxidant measures were assessed. Antioxidant levels were compared between men with and without a CVD outcome and all-cause mortality using independent samples t-tests. Lifestyle behaviours were compared across socioeconomic variables either by chi-square test for categorical variables or by Kruskal-Wallis one way ANOVA for continuous variables.

Cox proportional hazards models were used for analysis of antioxidant status for cardiovascular outcomes (first cardiovascular event) and separately for all-cause mortality, during a 10-year follow-up period. In the analysis of cardiovascular events, individuals were followed from baseline assessment to the first CVD event or death or end of followup. Each antioxidant was split into fourths using quartiles to categorise participants for analysis. The lowest quarter of the distribution of each antioxidant was used as the reference category for the calculation of hazard ratios. Firstly, models were run with minimal adjustment for potential confounders which included age and country, followed by a multivariate analysis that also included systolic blood pressure (SBP), diabetes, BMI, total cholesterol, high density lipoprotein (HDL) cholesterol, height, social class (low, medium and high), alcohol consumption (none, 1-128, 129-265, 266-461 and $\geq 462 \mathrm{~mL} /$ week), smoking status (never smoked, smoked other than cigarettes, smoked $<15$ cigarette pack years, smoked $\geq 15$ but $<30$ cigarette pack years and smoked $\geq 30$ cigarette pack years), and total physical activity (total physical activity was square root transformed, before inclusion in the model). Hazard ratios were compared across quarters of antioxidant distribution by a test for trend, and a likelihood ratio test was used to check for non-linearity by comparing the trend model with a model specifying a different hazard for each quarter. Additional sensitivity analyses considered the exclusion of variables that could potentially contribute to the causal pathway of CVD or all-cause mortality. Tests of the hazard proportionality assumption in the Cox model were performed using Schoenfeld residuals. All tests were conducted at the $p<0.05$ significance level using SPSS version 25 (IBM Corp, Armonk, NY, USA) and Stata release 14 (StatCorp, College Station, TX, USA). To check for possible effect modification, interactions between country and antioxidants were added to the Cox proportional hazards models. A more stringent level of significance was set at $p \leq 0.005$ to allow for multiple comparisons of potential interactions in the absence of any prior hypotheses.

\section{Results}

\section{Cardiovascular disease events}

Of the 10,600 PRIME study participants, 891 had a previous history of CVD at study entry (355 from NI and 536 from France) and were excluded from the analysis. The remaining 9709 men were followed up for 10 years (mean $=3506.81$ days) which equated to 93,281 person years. Follow-up was incomplete for 470 men (the number who were not known to have died and were not followed for 
the full 10 years), so the lost to follow-up rate was $4.8 \%$. Of 9709 men, there were 538 deaths from any cause and 440 fatal and non-fatal cardiovascular events after 10 years of follow-up. Population characteristics for those 9709 men are summarised according to CVD outcome and all-cause mortality (Table 1). Individuals who experienced a CVD outcome were on average older than men who had not ( 55.5 years versus 54.8 years), were more likely to be from Northern Ireland (38.4\% versus $24.0 \%$ ), to have diabetes (7.3\% versus $3.1 \%)$ and higher mean SBP $(141 \mathrm{mmHg}$ versus $133 \mathrm{mmHg}$ ). In addition, those who experienced a CVD outcome had higher mean BMI $\left(27.3 \mathrm{~kg} / \mathrm{m}^{2}\right.$ versus $26.5 \mathrm{~kg} /$ $\mathrm{m}^{2}$ ), higher mean total cholesterol $(2.30 \mathrm{~g} / \mathrm{L}$ versus $2.21 \mathrm{~g} / \mathrm{L})$ and median triglycerides $(1.44 \mathrm{~g} / \mathrm{L}$ versus $1.21 \mathrm{~g} / \mathrm{L})$ but lower mean HDL cholesterol $(0.45 \mathrm{~g} / \mathrm{L}$ versus $0.49 \mathrm{~g} / \mathrm{L})$, and height $(172.1 \mathrm{~cm}$ versus $172.8 \mathrm{~cm})$ and be less physically active (median METs 76.0 versus 83.2). Furthermore, they were more likely to be from the lowest social class $(31.0 \%$ versus $23.8 \%$ ), consume fewer daily FVJ portions (median 2.21 versus 2.43$)$, be heavy smokers $(31.3 \%$ versus $21.5 \%)$ and refrain from alcohol consumption (25.9\% versus $16.3 \%)$.

\section{Antioxidant concentrations}

Antioxidant status was available for 6961 participants and antioxidant quartile boundaries are shown in Supplementary

Table 1 Summary characteristics for cardiovascular disease outcomes and all-cause mortality for 9709 men from Northern Ireland and France who were free of cardiovascular disease at entry

\begin{tabular}{|c|c|c|c|c|c|c|}
\hline \multirow[t]{2}{*}{ Parameter } & \multicolumn{3}{|l|}{ CVD outcome } & \multicolumn{3}{|l|}{ All-cause mortality } \\
\hline & No & Yes & $p$ value & No & Yes & $p$ value \\
\hline Age (years), mean (SD) & $54.8(2.9)$ & $55.5(3.0)$ & $<0.001$ & $54.8(2.9)$ & $55.8(2.8)$ & $<0.001$ \\
\hline \multicolumn{7}{|l|}{ Country, $n(\%)$} \\
\hline Northern Ireland & $2221(24.0 \%)$ & $169(38.4 \%)$ & & $2219(24.2 \%)$ & $171(31.8 \%)$ & \\
\hline France & $7048(76.0 \%)$ & $271(61.6 \%)$ & $<0.001$ & $6952(75.8 \%)$ & $367(68.2 \%)$ & $<0.001$ \\
\hline \multicolumn{7}{|l|}{ Diabetes, $n(\%)$} \\
\hline Yes & $290(3.1 \%)$ & $32(7.3 \%)$ & & $287(3.1 \%)$ & $35(6.5 \%)$ & \\
\hline No & $8979(96.9 \%)$ & $408(92.7 \%)$ & $<0.001$ & $8884(96.9 \%)$ & $503(93.5 \%)$ & $<0.001$ \\
\hline Systolic blood pressure $(\mathrm{mmHg})$, mean (SD) & $133(19)$ & $141(22)$ & $<0.001$ & $133(19)$ & $139(22)$ & $<0.001$ \\
\hline Body mass index $\left(\mathrm{kg} / \mathrm{m}^{2}\right)$, mean $(\mathrm{SD})$ & $26.5(3.4)$ & $27.3(3.8)$ & $<0.001$ & $26.6(3.38)$ & $26.5(4.23)$ & 0.64 \\
\hline Total cholesterol (g/L), mean (SD) & $2.21(0.38)$ & $2.30(0.39)$ & $<0.001$ & $2.22(0.38)$ & $2.19(0.44)$ & 0.21 \\
\hline HDL cholesterol (g/L), mean (SD) & $0.49(0.13)$ & $0.45(0.12)$ & $<0.001$ & $0.49(0.13)$ & $0.49(0.15)$ & 0.72 \\
\hline Triglycerides (g/L), median (IQR) & $1.21(0.90-1.71)$ & $1.44(1.06-2.06)$ & $<0.001$ & $1.22(0.90-1.71)$ & $1.31(0.93-1.91)$ & 0.008 \\
\hline Height (cm), mean (SD) & $172.8(6.6)$ & $172.1(6.4)$ & 0.04 & $172.8(6.5)$ & $172.3(6.7)$ & 0.08 \\
\hline Total physical activity (MET),median (IQR) & $83.2(43-135)$ & $76.0(33.1-132)$ & 0.03 & $83.3(42.9-135)$ & $73.6(36.6-136)$ & 0.04 \\
\hline $\begin{array}{l}\text { Daily fruit, vegetable and juice portions, median } \\
\text { (IQR) }\end{array}$ & $2.43(1.64-3.36)$ & $2.21(1.39-3.00)$ & $<0.001$ & $2.43(1.64-3.36)$ & $2.14(1.39-3.14)$ & $<0.001$ \\
\hline \multicolumn{7}{|l|}{ Social class, $n(\%)$} \\
\hline Low & $2201(23.8 \%)$ & $136(31.0 \%)$ & & $2137(23.4 \%)$ & $200(37.3 \%)$ & \\
\hline Middle & $1396(15.1 \%)$ & $76(17.3 \%)$ & & $1403(15.4 \%)$ & $69(12.9 \%)$ & \\
\hline High & $5638(61.1 \%$ & $227(51.7 \%)$ & $<0.001$ & $5598(61.3 \%)$ & $267(49.8 \%)$ & $<0.001$ \\
\hline \multicolumn{7}{|l|}{ Alcohol, $n(\%)$} \\
\hline None & $1513(16.3 \%)$ & $114(25.9 \%)$ & & $1527(16.7 \%)$ & $100(18.6 \%)$ & \\
\hline $1-128 \mathrm{~mL} /$ week & $1997(21.5 \%)$ & $78(17.7 \%)$ & & $1983(21.6 \%)$ & $92(17.1 \%)$ & \\
\hline 129-265 mL/week & $1981(21.4 \%)$ & $92(20.9 \%)$ & & $1974(21.5 \%)$ & $99(18.4 \%)$ & \\
\hline 266-461 mL/week & $1873(20.2 \%)$ & $71(16.1 \%)$ & & $1845(20.1 \%)$ & $99(18.4 \%)$ & \\
\hline$\geq 462 \mathrm{~mL} /$ week & $1905(20.6 \%)$ & $85(19.3 \%)$ & $<0.001$ & $1842(20.1 \%)$ & $148(27.5 \%)$ & $<0.001$ \\
\hline \multicolumn{7}{|l|}{ Smoking, $n(\%)$} \\
\hline Never & $2763(30.0 \%)$ & $111(25.5 \%)$ & & $2765(30.3 \%)$ & $109(20.3 \%)$ & \\
\hline Smoked other than cigarettes & $702(7.6 \%)$ & $27(6.2 \%)$ & & $696(7.6 \%)$ & $33(6.2 \%)$ & \\
\hline Smoked $<15$ pack years & $2008(21.8 \%)$ & $66(15.2 \%)$ & & $1999(21.9 \%)$ & $75(14.0 \%)$ & \\
\hline Smoked $\geq 15$ but $<30$ pack years & $1755(19.1 \%)$ & $95(21.8 \%)$ & & $1750(19.2 \%)$ & $100(18.7 \%)$ & \\
\hline Smoked $\geq 30$ pack years & $1984(21.5 \%)$ & $136(31.3 \%)$ & $<0.001$ & $1901(20.9 \%)$ & $219(40.9 \%)$ & $<0.001$ \\
\hline
\end{tabular}

$M E T$ metabolic equivalent of task (per week), $S D$ standard deviation, $I Q R$ interquartile range 
Table 1. As expected, there was a high correlation between fruit and vegetable consumption and antioxidant measures (Supplementary Table 2). Over a third of participants (37.6\%) had plasma vitamin $\mathrm{C}$ concentrations considered to be optimum for health $(>50 \mu \mathrm{mol} / \mathrm{L})$, over three quarters (77.6\%) had plasma vitamin $\mathrm{C}$ concentrations above the marginal deficiency level $(>22.7 \mu \mathrm{mol} / \mathrm{L})$, and $9.2 \%$ of participants had concentrations considered extremely deficient $(<11 \mu \mathrm{mol} / \mathrm{L})$ [28-30] (data not shown). For $\alpha$-tocopherol, severe overt deficiency has been reported at $11.6 \mu \mathrm{mol} / \mathrm{L}$ [28] and levels $>27.5 \mu \mathrm{mol} / \mathrm{L}$ have been considered optimum to reduce the risk associated with ischaemic heart disease (IHD) [29]. More than two fifths of participants (40.5\%) had plasma alpha tocopherol concentrations recommended for optimum health $(>27.5 \mu \mathrm{mol} / \mathrm{L})$. Almost all participants $(99.7 \%$ ) had alpha tocopherol concentrations in excess of the overt deficiency value of $11.6 \mu \mathrm{mol} / \mathrm{L}$ (data not shown).

Optimum recommended concentrations to reduce IHD risk have previously been proposed for retinol $(\geq 2.2 \mu \mathrm{mol} / \mathrm{L})$ and $\beta$-carotene $(>0.4 \mu \mathrm{mol} / \mathrm{L})$ [28-30]. Over half of the participants $(54.5 \%)$ had plasma retinol concentrations $\geq 2.2 \mu \mathrm{mol} / \mathrm{L}$ and over one third $(36.2 \%)$ had a $\beta$-carotene concentration $>0.4 \mu \mathrm{mol} / \mathrm{L}$.

\section{Cardiovascular disease outcome}

With the exception of both $\alpha$ - and $\gamma$-tocopherol, lower levels of all antioxidants were detected in men who experienced a CVD outcome, although this was not statistically significant for zeaxanthin and retinol (Table 2). Hazard ratios in a model minimally adjusted for age and country, showed a significant reduction in risk across quarters for alpha and beta carotene, vitamin C, lycopene, $\alpha$-tocopherol and mean SAS $(p<0.05$; Table 3). Following further adjustment for SBP, diabetes, BMI, cholesterol, HDL cholesterol, triglycerides, height, total physical activity, alcohol consumption, smoking and socioeconomic status, significant reductions across quarters of cardiovascular event risk were only observed for retinol, with $\alpha$ - and $\beta$-carotene falling just short of the significance threshold ( $p<0.05$; Table 4, Fig. 1$)$. The mean SAS divided in quarters showed no significant association with CVD outcomes in the fully adjusted model (Table 4). Associations with CVD outcomes were little altered by the omission of total cholesterol, HDL cholesterol, triglycerides, and systolic blood pressure with the exception of the attenuated effect on retinol that no longer remained significant (Supplementary Tables $3 \mathrm{a}$ and $3 \mathrm{~b}$ ).

\section{All-cause mortality outcome}

Men who died during follow-up were on average older (55.8 years versus 54.8 years), more likely to be from NI ( $31.8 \%$ versus $25.2 \%)$ and twice as likely to have diabetes (6.5\% versus $3.1 \%$ ) than men who survived. They also had higher mean SBP (139 $\mathrm{mmHg}$ versus $133 \mathrm{mmHg}$ ), higher median triglyceride levels $(1.31 \mathrm{~g} / \mathrm{L}$ versus $1.22 \mathrm{~g} / \mathrm{L})$ and were less physically active (median METs 73.6 versus 83.3). Furthermore, they were significantly more likely to be from the low social class (37.3\% versus $23.4 \%$ ), to consume fewer daily FVJ portions (median 2.14 versus 2.43 ) and have higher levels of both smoking and alcohol consumption $(p<0.001)$.

There were lower levels of all antioxidants associated with all-cause mortality, and only that of $\gamma$-tocopherol was not significant (Table 2). Similarly fully adjusted hazard ratios showed statistically significant reductions for all antioxidants in association with all-cause mortality, with the exception of $\gamma$-tocopherol (Fig. 1; Tables 3 and 4). Daily FVJ portions were initially significant when fitted with other confounders within the adjusted model but was no longer significant when the mean $Z$ score was included. The mean SAS showed significantly reduced risk across quarters for

Table 2 Geometric mean antioxidant levels and interquartile range (IQR) for cardiovascular disease outcomes and all-cause mortality for 6961 men from Northern Ireland and France who were free of cardiovascular disease at entry

\begin{tabular}{|c|c|c|c|c|c|c|c|}
\hline \multirow{2}{*}{$\begin{array}{l}\text { Antioxidant } \\
\mu \mathrm{mol} / \mathrm{L}\end{array}$} & \multicolumn{4}{|l|}{ CVD outcome } & \multicolumn{3}{|c|}{ All-Cause Mortality } \\
\hline & All $(n=6961)$ & No $(n=6747)$ & Yes $(n=214)$ & $p$ value & No $(n=6545)$ & Yes $(n=358)$ & $p$ value \\
\hline Vitamin C & $33.8(24.8-59.2)$ & $33.9(25.0-59.2)$ & $29.8(19.4-57.8)$ & 0.045 & $34.4(25.5-59.4)$ & $24.1(15.1-55.0)$ & $<0.001$ \\
\hline Lycopene & $0.67(0.42-1.34)$ & $0.67(0.42-1.35)$ & $0.52(0.31-1.09)$ & 0.001 & $0.68(0.43-1.35)$ & $0.48(0.27-1.08)$ & $<0.001$ \\
\hline$\beta$-Carotene & $0.29(0.17-0.51)$ & $0.29(0.17-0.51)$ & $0.22(0.11-0.39)$ & $<0.001$ & $0.29(0.18-0.52)$ & $0.20(0.11-0.40)$ & $<0.001$ \\
\hline$\alpha$-Carotene & $0.11(0.07-0.19)$ & $0.11(0.07-0.19)$ & $0.08(0.05-0.15)$ & $<0.001$ & $0.11(0.07-0.20)$ & $0.08(0.05-0.15)$ & $<0.001$ \\
\hline$\beta$-Cryptoxanthin & $0.70(0.04-0.10)$ & $0.07(0.04-0.10)$ & $0.06(0.04-0.09)$ & 0.008 & $0.07(0.04-0.11)$ & $0.05(0.03-0.08)$ & $<0.001$ \\
\hline Zeaxanthin & $0.04(0.03-0.06)$ & $0.04(0.03-0.06)$ & $0.04(0.03-0.06)$ & 0.125 & $0.04(0.03-0.06)$ & $0.04(0.02-0.05)$ & $<0.001$ \\
\hline Lutein & $0.21(0.16-0.31)$ & $0.22(0.16-0.31)$ & $0.19(0.14-0.30)$ & 0.006 & $0.22(0.16-0.31)$ & $0.17(0.12-0.27)$ & $<0.001$ \\
\hline$\alpha$-Tocophorol & $26.0(22.2-30.4)$ & $25.9(22.1-30.4)$ & $26.8(22.5-31.5)$ & 0.059 & $26.0(22.3-30.5)$ & $24.6(20.5-29.8)$ & $<0.001$ \\
\hline$\gamma$-Tocophorol & $2.35(1.88-2.95)$ & $2.35(1.88-2.95)$ & $2.40(1.93-2.96)$ & 0.375 & $2.35(1.88-2.95)$ & $2.31(1.84-2.86)$ & 0.422 \\
\hline Retinol & $2.24(1.92-2.64)$ & $2.24(1.92-2.64)$ & $2.22(1.87-2.59)$ & 0.525 & $2.24(1.93-2.64)$ & $2.15(1.83-2.57)$ & 0.006 \\
\hline
\end{tabular}


Table 3 Minimally adjusted for age and country Cox Proportional Hazard Ratios (HR) by quartile for cardiovascular disease outcomes and allcause mortality

\begin{tabular}{|c|c|c|c|c|c|c|c|c|}
\hline & \multicolumn{4}{|l|}{ CVD outcomes } & \multicolumn{4}{|c|}{ All-cause mortality } \\
\hline & $\begin{array}{l}\text { Q2 HR (95\% } \\
\text { CI) }\end{array}$ & $\begin{array}{l}\text { Q3 HR }(95 \% \\
\text { CI) }\end{array}$ & $\begin{array}{l}\text { Q4 HR (95\% } \\
\text { CI) }\end{array}$ & $P$ & $\begin{array}{l}\text { Q2 HR (95\% } \\
\text { CI) }\end{array}$ & $\begin{array}{l}\text { Q3 HR }(95 \% \\
\text { CI) }\end{array}$ & $\begin{array}{l}\text { Q4 HR (95\% } \\
\text { CI) }\end{array}$ & $p$ \\
\hline Vitamin C & $0.77(0.54-1.11)$ & $0.59(0.40-0.87)$ & $0.72(0.50-1.04)$ & 0.04 & $0.57(0.43-0.75)$ & $0.49(0.37-0.66)$ & $0.51(0.39-0.68)$ & $<0.001$ \\
\hline Lycopene & $0.86(0.61-1.23)$ & $0.79(0.55-1.13)$ & $0.56(0.38-0.85)$ & 0.006 & $0.69(0.53-0.90)$ & $0.53(0.40-0.71)$ & $0.51(0.38-0.68)$ & $<0.001$ \\
\hline$\beta$-Carotene & $0.59(0.41-0.85)$ & $0.77(0.55-1.09)$ & $0.40(0.26-0.62)$ & $<0.001$ & $0.59(0.46-0.77)$ & $0.55(0.42-0.72)$ & $0.36(0.26-0.49)$ & $<0.001$ \\
\hline$\alpha$-Carotene & $0.85(0.60-1.20)$ & $0.64(0.44-0.93)$ & $0.54(0.36-0.82)$ & 0.001 & $0.79(0.62-1.02)$ & $0.56(0.42-0.74)$ & $0.40(0.29-0.56)$ & $<0.001$ \\
\hline$\beta$-Cryptoxanthin & $0.85(0.60-1.23)$ & $0.81(0.56-1.18)$ & $0.67(0.45-1.01)$ & 0.06 & $0.39(0.29-0.52)$ & $0.47(0.36-0.62)$ & $0.30(0.22-0.41)$ & $<0.001$ \\
\hline Zeaxanthin & $0.97(0.66-1.41)$ & $0.87(0.57-1.31)$ & $1.02(0.68-1.54)$ & 0.97 & $0.48(0.36-0.63)$ & $0.45(0.34-0.60)$ & $0.36(0.26-0.49)$ & $<0.001$ \\
\hline Lutein & $0.77(0.53-1.13)$ & $0.77(0.51-1.16)$ & $0.78(0.51-1.19)$ & 0.27 & $0.67(0.51-0.87)$ & $0.48(0.36-0.65)$ & $0.40(0.29-0.55)$ & $<0.001$ \\
\hline$\alpha$-Tocophorol & $0.84(0.56-1.28)$ & $1.21(0.83-1.77)$ & $1.32(0.91-1.91)$ & 0.049 & $0.53(0.40-0.70)$ & $0.65(0.49-0.85)$ & $0.62(0.47-0.81)$ & 0.002 \\
\hline$\gamma$-Tocophorol & $1.17(0.79-1.73)$ & $1.25(0.85-1.84)$ & $1.14(0.77-1.69)$ & 0.50 & $0.83(0.63-1.10)$ & $0.88(0.67-1.16)$ & $0.78(0.59-1.04)$ & 0.13 \\
\hline Retinol & $0.81(0.56-1.19)$ & $0.87(0.60-1.26)$ & $0.85(0.58-1.23)$ & 0.45 & $0.71(0.54-0.94)$ & $0.75(0.57-0.98)$ & $0.77(0.58-1.01)$ & 0.07 \\
\hline Mean SAS & $0.77(0.56-1.08)$ & $0.68(0.48-0.97)$ & $0.67(0.47-0.96)$ & 0.02 & $0.52(0.41-0.67)$ & $0.36(0.27-0.48)$ & $0.37(0.28-0.49)$ & $<0.001$ \\
\hline
\end{tabular}

THR indicated for quarters 2,3 and 4 are relative to the reference quarter 1

$Q$ quarter, $H R$ hazard ratio, $C I$ confidence intervals, Mean SAS mean standardised antioxidant score, $p p$ for trend

Table 4 Adjusted Cox Proportional Hazard Ratios (HR) by quartile for cardiovascular disease outcomes and all-cause mortality

\begin{tabular}{|c|c|c|c|c|c|c|c|c|}
\hline & \multicolumn{4}{|l|}{ CVD outcomes } & \multicolumn{4}{|c|}{ All-cause mortality } \\
\hline & $\begin{array}{l}\text { Q2 HR (95\% } \\
\text { CI) }\end{array}$ & $\begin{array}{l}\text { Q3 HR (95\% } \\
\text { CI) }\end{array}$ & $\begin{array}{l}\text { Q4 HR (95\% } \\
\text { CI) }\end{array}$ & $p$ & $\begin{array}{l}\text { Q2 HR (95\% } \\
\text { CI) }\end{array}$ & $\begin{array}{l}\text { Q3 HR (95\% } \\
\text { CI) }\end{array}$ & $\begin{array}{l}\text { Q4 HR (95\% } \\
\text { CI) }\end{array}$ & $p$ \\
\hline Vitamin C & $0.85(0.59-1.24)$ & $0.69(0.46-1.03)$ & $0.86(0.58-1.26)$ & 0.27 & $0.69(0.52-0.92)$ & $0.63(0.46-0.84)$ & $0.66(0.49-0.89)$ & 0.003 \\
\hline Lycopene & $0.93(0.64-1.34)$ & $0.95(0.65-1.38)$ & $0.73(0.47-1.14)$ & 0.24 & $0.77(0.59-1.00)$ & $0.63(0.47-0.85)$ & $0.70(0.51-0.96)$ & 0.005 \\
\hline$\beta$-Carotene & $0.61(0.42-0.90)$ & $0.90(0.62-1.30)$ & $0.54(0.34-0.86)$ & 0.06 & $0.71(0.54-0.93)$ & $0.71(0.53-0.95)$ & $0.53(0.37-0.74)$ & $<0.001$ \\
\hline$\alpha$-Carotene & $0.92(0.65-1.32)$ & $0.73(0.49-1.09)$ & $0.71(0.45-1.12)$ & 0.07 & $0.92(0.71-1.20)$ & $0.72(0.53-0.96)$ & $0.56(0.40-0.80)$ & 0.001 \\
\hline$\beta$-Cryptoxanthin & $0.94(0.64-1.36)$ & $0.99(0.67-1.47)$ & $0.86(0.56-1.32)$ & 0.57 & $0.46(0.35-0.62)$ & $0.63(0.47-0.83)$ & $0.41(0.29-0.57)$ & $<0.001$ \\
\hline Zeaxanthin & $1.02(0.69-1.51)$ & $1.00(0.65-1.54)$ & $1.26(0.81-1.95)$ & 0.36 & $0.58(0.43-0.77)$ & $0.60(0.44-0.81)$ & $0.50(0.36-0.71)$ & $<0.001$ \\
\hline Lutein & $0.81(0.55-1.19)$ & $0.91(0.59-1.38)$ & $0.90(0.57-1.41)$ & 0.73 & $0.79(0.60-1.04)$ & $0.63(0.46-0.86)$ & $0.56(0.40-0.79)$ & $<0.001$ \\
\hline$\alpha$-Tocophorol & $0.85(0.56-1.30)$ & $1.13(0.75-1.68)$ & $0.99(0.64-1.53)$ & 0.72 & $0.56(0.42-0.75)$ & $0.68(0.51-0.91)$ & $0.66(0.48-0.91)$ & 0.02 \\
\hline$\gamma$-Tocophorol & $1.06(0.70-1.59)$ & $1.14(0.76-1.70)$ & $0.90(0.59-1.38)$ & 0.69 & $0.87(0.65-1.17)$ & $1.01(0.76-1.35)$ & $0.83(0.61-1.13)$ & 0.43 \\
\hline Retinol & $0.82(0.56-1.21)$ & $0.76(0.52-1.12)$ & $0.65(0.43-0.98)$ & 0.04 & $0.70(0.53-0.94)$ & $0.74(0.56-0.99)$ & $0.68(0.50-0.91)$ & 0.02 \\
\hline Mean SAS & $0.83(0.59-1.17)$ & $0.79(0.55-1.14)$ & $0.76(0.51-1.13)$ & 0.16 & $0.61(0.47-0.79)$ & $0.46(0.34-0.62)$ & $0.50(0.37-0.68)$ & $<0.001$ \\
\hline
\end{tabular}

The HR indicated for quarters 2, 3 and 4 are relative to the reference quarter 1. The Cox Proportional HRs are adjusted for age, country, diabetes, alcohol and smoking status, systolic blood pressure, body mass index, total cholesterol, high density lipoprotein cholesterol, triglycerides, height, social class and physical activity level

$Q$ quarter, $H R$ hazard ratio, $C I$ confidence intervals, Mean SAS mean standardised antioxidant score, $p p$ for trend

all-cause mortality in minimally and fully adjusted models (Tables 3 and $4 ; p<0.001$ ). Lack of linearity was observed for the associations between mortality and $\alpha$-tocopherol, $\beta$-cryptoxanthin, zeaxanthin and SAS. Similar adjusted Cox proportional HR by quartile for smoking-associated cancer and all neoplasm-related mortality according to ICD Ninth Revision codes are presented in Supplementary Table 4.

No significant deviations from the tests of the hazard proportionality assumption in the Cox model using Schoenfeld residuals were detected, for either CVD events or all-cause mortality. There was no evidence of significant interaction between country and antioxidants in any of the models for CVD events or all-cause mortality following correction for multiple testing. 


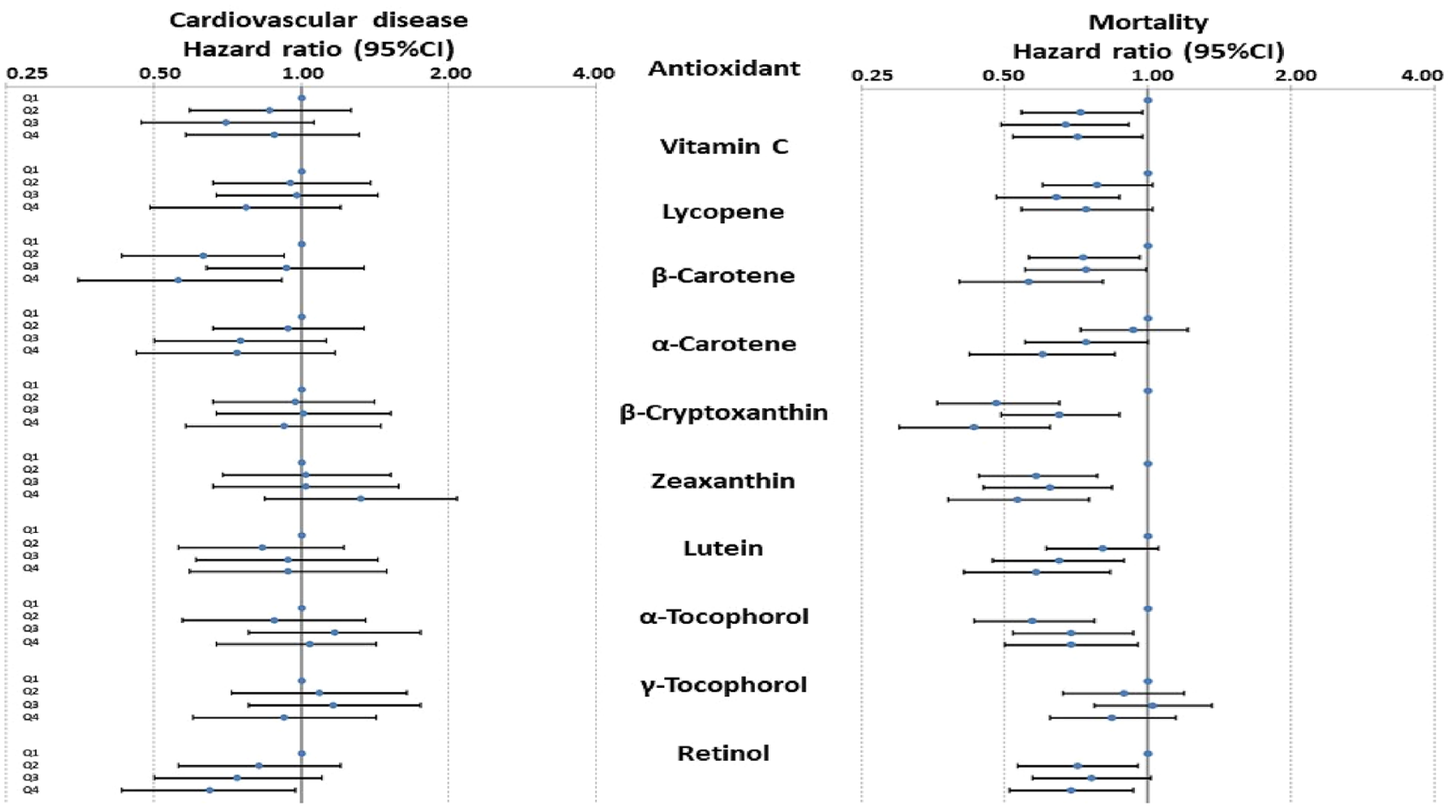

Fig. 1 Hazard ratios for death from all causes and for a cardiovascular event by quarters of antioxidant levels, adjusting for age, country, diabetes, alcohol and smoking status, systolic blood pressure, body

\section{Discussion}

In this study, we observed associations between lower concentrations of serum micronutrients vitamin $\mathrm{C}$, retinol, $\alpha$-tocopherol, and six carotenoids, including lycopene, $\alpha$-carotene, $\beta$-carotene, $\beta$-cryptoxanthin, zeaxanthin and lutein, and all-cause mortality over a 10-year follow-up period, using data from a large cohort of 9709 predominantly white, middle-aged men who were free from CVD at the time of recruitment in NI and France. Furthermore, higher plasma retinol concentrations were also significantly associated with a lower risk of CVD events after adjustment for potential confounding variables.

Oxidative stress and systemic inflammatory damage have been implicated in the aetiology of several chronic diseases, including CVD [2-4], with nutritional influences previously estimated to contribute to almost $45 \%$ of all cardiometabolic deaths in the United States in 2012 [5-10]. Previously, we reported low plasma retinol independent of carotenoid levels in association with CVD risk, in a nested case control study of PRIME participants after 5-year follow-up [5]. Retinol is partly replaced by its major precursor, $\beta$-carotene, although conversion is poor and inconsistent [31]. The relatively moderate association of plasma retinol in the present evaluation may be limited by the complexities of plasma retinol determination, dietary influences and vitamin A status, mass index, total cholesterol, high density lipoprotein cholesterol, triglycerides, height, social class and physical activity level

especially given the influences of hepatic homeostasis and reported age-dependent variation in middle-aged westernised males, mean age at sampling/recruitment of 55 years, and subsequent CVD and all-cause mortality outcomes over the following decade [5, 31]. All other evaluated micronutrients were not significantly associated with reduced CVD risk, following adjustment for potential confounders. These findings largely support previous observational reports of inverse associations between all-cause mortality, cardiovascular risk and serum micronutrient status [5, 32, 33], supported by higher fruit and vegetable consumption and antioxidant intake [34-36].

Carotenoids are lipophilic phytochemicals represented by two sub-classes based upon their polarity: non-polar carotenes include $\alpha$-carotene, $\beta$-carotene and lycopene while polar xanthophylls include lutein, zeaxanthin and $\beta$-cryptoxanthin. The individual carotenoid properties are dependent on their unique functional group and the conjugated double-bonding present [37]. Carotenoids have been reported to reduce the detrimental impact of oxidative stressinduced diseases including CVD, given the antioxidant and anti-inflammatory properties that result from their chemical structure and interaction with biological membranes [38]. Interestingly, the reduced risk of CVD events and all-cause mortality associated with antioxidants identified from observational epidemiological studies has not been observed in 
supplementation trials, some of which have not only failed to show any health benefits but have reported unanticipated harmful effects [14]. Huang and colleagues also recently identified inverse associations between serum $\beta$-carotene and all-cause mortality, including CVD related deaths in a prospective serological analysis of 29,103 men in the ATBC study (Alpha-Tocopherol, Beta-Carotene Cancer Prevention) over 31 years of follow-up that assessed 23,796 deaths [14]. Randomised supplementation trials have been largely limited to single antioxidant studies, in contrast to observational epidemiological investigations of plasma antioxidant levels or dietary intake of foods rich in these nutrients. The dietary origins of these antioxidants (fruits, vegetables, berries, etc.) also contain additional natural compounds that may enhance the micronutrient properties that are difficult to replicate in the context of supplementation studies [10]. Previous investigations have highlighted that individuals with low micronutrient status are more likely to benefit from randomised supplementation trials [15]. Reduced all-cause mortality was reported previously following supplementation in nutritionally deficient individuals with low plasma antioxidant levels, but excess levels in sufficient individuals may not provide additional benefit and result in potentially toxic effects [15-17].

$\beta$-Carotene is one of the most widely studied carotenoids given its abundance in fruit and vegetables and its provitamin A activity. The beneficial antioxidant properties of $\beta$-carotene include improved immunological function through enhanced lymphocyte proliferation that reduces LDL susceptibility to oxidative modification [39]. Nevertheless, the benefits associated with dietary consumption appear to be lost when it is provided by pharmacological supplementation, which in turn may result in harmful effects in some sub-populations, such as smokers, where increased incidence of lung cancer and CVD have been reported previously [40]. A possible explanation for the lack of association between serum micronutrient status and CVD beyond retinol, may result from the lower number of recorded CVD events than deaths in PRIME participants (440 versus 538), and as blood samples were not available for all PRIME participants, the number of CVD events included in the analysis was further limited, which may have reduced statistical power. Survival bias may also have been a factor, as men with lower plasma micronutrient concentrations would be more likely to have died prematurely during the 10-year follow-up period and therefore were less likely to have recorded a CVD event within the specified time period, given the resultant death.

Higher plasma concentrations of vitamin C, retinol, $\alpha$-tocopherol and all carotenoids were associated with reduced all-cause mortality among the middle-aged male participants of the PRIME study. In keeping with the findings reported here, a recent systematic review and meta-analyses identified no associations between $\alpha$-carotene, lycopene, $\beta$-cryptoxanthin, lutein and zeaxanthin with cardiovascular outcomes [10]. Aune and colleagues meta-analyses reported associations between $\alpha$-carotene, lycopene, $\beta$-cryptoxanthin and mortality similar to our findings, although in contrast, they failed to detect associations between lutein and zeaxanthin and overall mortality [10]. The limited statistical power associated with the analysis of incident CVD cases reported in the current study and the small number of studies available for meta-analyses previously, should be considered in the context of the findings reported.

In contrast to the other micronutrients evaluated in the current study, plasma $\gamma$-tocopherol levels were not associated with all-cause mortality. Evaluation of the effects of vitamin E has predominately focused on $\alpha$-tocopherol, with evidence specific to $\gamma$-tocopherol and health-related outcomes, less commonly studied [41]. Previous studies, such as the British National Diet and Nutrition Survey of those aged 65 years and over, also found no evidence of association between plasma $\gamma$-tocopherol and overall mortality (HR 0.96, $p=0.3$ ) [42]. In contrast, the Multiethnic Cohort Study evaluated 1489 deaths reported in 8365 participants and serum $\gamma$-tocopherol was positively associated with all-cause, cancer and CVD mortality in adjusted models [32].

The potential benefits of vitamin $\mathrm{C}$ on mortality have been previously reported [43] and several potential mechanisms proposed, including a protective effect on the vascular endothelium, preventing uncontrolled proliferation of vascular smooth muscle cells and inhibition of pro-inflammatory cytokines and adhesion molecules central to the atherogenic processes [44]. Previous studies have also reported associations between higher carotenoid concentrations and lower cancer [7, 45], dementia [46] and CVD risk [5-10] and between carotenoids and markers of inflammation and oxidative stress [13].

These findings have important implications for future antioxidant research, given the variation in effects on allcause mortality observed, highlighting the importance of measuring individual micronutrient levels. Antioxidants possess different molecular properties and biological activities that confer variable health benefits and the non-linear relationship observed for some antioxidants suggest a beneficial range that may be more readily achieved through dietary interventions as opposed to high-dose supplementation [15]. Micronutrients are unlikely to act independently in their reduction of mortality risk but reflect specific food groups, such as fruit and vegetables, that are associated with improved health outcomes [47]. Further assessment of the interactions, optimal concentrations and potential health benefits of antioxidants will improve the design of dietary interventions and identify those most likely to benefit. 


\section{Strengths and limitations of this study}

The major strengths of our study were the large number of well-characterised participants with prospective follow-up over 10 years and clinically validated cardiovascular events. Many observational studies are restricted by small sample size and/or short follow-up duration, limiting the reliability of the associations reported. Associations were based only on those men free from cardiovascular events at study entry. In addition, the micronutrient plasma measurements were quantified using standardised and validated laboratory methods, and so provided an objective determinant, independent of dietary intake estimates recalled over a period of time. Assessment of antioxidant concentrations as continuous variables, identified similar associations to the evaluation of antioxidants by quartiles but the latter is more amenable to the identification of non-linear relationships, where they exist. Alpha-carotene and lutein were the only antioxidants that demonstrated a dose-response relationship with allcause mortality, with higher concentrations associated with lower mortality. Retinol was the only antioxidant significantly associated with CVD events demonstrating a linear relationship with reduced risk with higher concentrations. A mean SAS offers an opportunity to assess the cumulative effects of antioxidant concentrations on CVD outcomes and all-cause mortality. Although a combined total antioxidant score was more strongly associated with all-cause mortality than individual antioxidants, the effects observed were not substantially different than for individual antioxidants alone.

Our study has several limitations. We tested multiple hypotheses and, as such, our results must be interpreted with caution as we made no correction for multiple testing in the analyses presented in Tables 2, 3 and 4, although several of the reported associations would remain significant even if tested at the $p<0.001$ level. Despite controlling for the most important covariates, additional residual confounding may exist. Furthermore, additional dietary factors not considered within the analyses may be associated with antioxidant levels. Moreover, the sensitivity of nutritional biomarkers is somewhat limited by the influences of individual genetic variation associated with dietary intake and nutrient metabolism, a complex multistage process that includes ingestion, digestion, absorption and transportation in the blood [48, 49]. The categorical analyses by quartiles depends on certain parametric modelling assumptions (proportional hazards, linearity, etc.), although analyses of antioxidant levels as continuous variables demonstrated similar levels of significance (data not shown). In addition, while we evaluated daily FVJ consumption, the use of FFQ data may lead to an overestimation of the intakes of some healthy foods, which may impact the interpretation of the associations observed, although this had little effect on the associated hazard ratio estimates. Furthermore, we used a single measure of plasma antioxidant levels which may not necessarily reflect longterm nutritional status against CVD and all-cause mortality outcomes. Previous analysis of The Third National Health and Nutrition Examination Survey (NHANES III) investigated 4225 deaths in 16,008 participants over a median 14.2 years follow-up period and reported that a single serum measurement likely represents a reliable estimate of longterm antioxidant status [15]. Additionally, associations between micronutrient status and fruit and vegetable intake were strong, supporting the plasma concentration as an appropriate representative measure. Although we examined the independent and cumulative effects of ten antioxidants on cardiovascular events and all-cause mortality, potential associations with other nutrients such as omega-3-fatty acids, vitamin $\mathrm{D}$, the $\mathrm{B}$-vitamins and selenium were beyond the scope of this study. Finally, our study results apply to predominantly white European middle-aged men who were initially free from CVD at the point of recruitment.

In summary, we have shown that higher plasma concentrations of vitamin $\mathrm{C}$, retinol, $\alpha$-tocopherol and six carotenoids ( $\alpha$-carotene, $\beta$-carotene, $\beta$-cryptoxanthin, lutein, lycopene, zeaxanthin) were associated with a lower risk of all-cause mortality in middle-aged men. Furthermore, higher levels of retinol were associated with reduced risk of CVD events. Our findings are of major public health significance as the search for improved understanding of the modifiable risk factors associated with cardiovascular events and allcause mortality continues. Identifying individuals at future increased risk of developing CVD and detecting adverse risk at the earliest stages, may be most effective in delaying disease progression and preventing premature death. Our findings suggest that, at a population level, small, achievable shifts in micronutrient status could have a considerable impact on mortality and CVD risk. However, there is still insufficient evidence to establish a causal relationship between low antioxidant levels and all-cause mortality. Given the potential implications of nutritional status in the aetiology of multiple chronic conditions, dietary intervention warrants further investigation and may prove beneficial in reducing premature mortality, especially given the largely null findings from randomised control trails of antioxidant supplementation. Insufficient consideration for individual participant baseline nutrition levels within study inclusion criteria, may represent a major concern for randomised trial design. Our findings suggest individuals deficient in specific micronutrients may derive greater benefit from dietary or supplement interventions than sufficient individuals.

Acknowledgements We acknowledge the contributions from the PRIME study participants over the duration of the investigation period. The PRIME Study was organised under an agreement between INSERM and the Merck, Sharpe and Dohme-Chibret Laboratory, with the following participating Laboratories: The Strasbourg MONICA Project, Strasbourg, France (D. Arveiler, B. Haas). The Toulouse 
MONICA Project, INSERM U558, Toulouse, France (J. Ferrières, JB. Ruidavets). The Lille MONICA Project, INSERM U744, Lille, France (P. Amouyel, M. Montaye). The Department of Epidemiology and Public Health, Queen's University Belfast, NI (A. Evans, J. Yarnell, F. Kee). The Department of Atherosclerosis, INSERM U545, Lille, France (G. Luc, JM. Bard). The Laboratory of Hematology, La Timone Hospital, Marseille, France (I. Juhan-Vague). The Laboratory of Endocrinology, INSERM U326, Toulouse, France (B. Perret). The Vitamin Research Unit, The University of Bern, Bern, Switzerland (F. Gey). The Trace Element Laboratory, Department of Medicine, Queen's University Belfast, NI (J. Woodside, I. Young). The DNA Bank, INSERM U525, Paris, France (F. Cambien). The Coordinating Center, INSERM U909, Villejuif, France (P. Ducimetière, A. Bingham). The PRIME Study is supported by grants from INSERM, Merck, Sharpe and Dohme-Chibret Laboratory, the French Research Agency and the Foundation Heart and Arteries. We also acknowledge the contribution of the members of the event validation Committees: Pr L. Guize (deceased), Dr C. Morrison, Dr M-T. Guillanneuf, Pr M. Giroud; and the Alliance Partnership Programme for its financial support.

Author contributions JVW conceived the experiments; LN performed the experiments; GJM and CCP analysed the data; GJM, CCP and JVW wrote the manuscript. All authors reviewed and approved the final version of the manuscript. GJM is the guarantor of this work and, as such, had full access to all the data in the study and takes responsibility for the integrity and accuracy of the data.

\section{Compliance with ethical standards}

Conflict of interest All authors declare no competing financial interests.

Open Access This article is licensed under a Creative Commons Attribution 4.0 International License, which permits use, sharing, adaptation, distribution and reproduction in any medium or format, as long as you give appropriate credit to the original author(s) and the source, provide a link to the Creative Commons licence, and indicate if changes were made. The images or other third party material in this article are included in the article's Creative Commons licence, unless indicated otherwise in a credit line to the material. If material is not included in the article's Creative Commons licence and your intended use is not permitted by statutory regulation or exceeds the permitted use, you will need to obtain permission directly from the copyright holder. To view a copy of this licence, visit http://creativecommons.org/licenses/by/4.0/.

\section{References}

1. WHO. https://www.who.int/news-room/fact-sheets/detail/cardi ovascular-diseases-(cvds). Accessed 1 July 2020

2. Libby $\mathbf{P}$ (2017) Interleukin-1 beta as a target for atherosclerosis therapy: biological basis of CANTOS and beyond. J Am Coll Cardiol 70:2278-2289

3. Libby P, Hansson GK (2018) Taming immune and inflammatory responses to treat atherosclerosis. J Am Coll Cardiol 71:173-176

4. Ceriello A, Motz E (2004) Is oxidative stress the pathogenic mechanism underlying insulin resistance, diabetes, and cardiovascular disease? The common soil hypothesis revisited. ArteriosclerThrombVasc Biol 24:816-823

5. Gey KF, Ducimetière P, Evans A, Amouyel P, Arveiler D, Ferrières J, Luc G, Kee F, Bingham A, Yarnell J, Cambien F (2010) Low plasma retinol predicts coronary events in healthy middleaged men: the PRIME study. Atherosclerosis 208:270-274
6. Woodside JV, Yarnell JW, Patterson CC, Arveiler D, Amouyel P, Ferrières J, Kee F, Evans A, Bingham A, Ducimetière P (2012) Do lifestyle behaviours explain socioeconomic differences in allcause mortality, and fatal and non-fatal cardiovascular events? Evidence from middle aged men in France and Northern Ireland in the PRIME Study. Prev Med 54:247-253

7. Aune D, Giovannucci E, Boffetta P, Fadnes LT, Keum N, Norat T, Greenwood DC, Riboli E, Vatten LJ, Tonstad S (2017) Fruit and vegetable intake and the risk of cardiovascular disease, total cancer and all-cause mortality - a systematic review and doseresponse meta-analysis of prospective studies. Int J Epidemiol 46:1029-1056

8. Micha R, Peñalvo JL, Cudhea F, Imamura F, Rehm CD, Mozaffarian D (2017) Association between dietary factors and mortality from heart disease, stroke, and type 2 diabetes in the United States. JAMA 317:912-924

9. Picó C, Serra F, Rodríguez AM, Keijer J, Palou A (2019) Biomarkers of nutrition and health: new tools for new approaches. Nutrients 11:1092

10. Aune D, Keum N, Giovannucci E, Fadnes LT, Boffetta P, Greenwood DC, Tonstad S, Vatten LJ, Riboli E, Norat T (2018) Dietary intake and blood concentrations of antioxidants and the risk of cardiovascular disease, total cancer, and all-cause mortality: a systematic review and dose-response meta-analysis of prospective studies. Am J ClinNutr 108:1069-1091

11. Steinberg D, Parthasarathy S, Carew TE, Khoo JC, Witztum JL (1989) Beyond cholesterol. Modifications of low-density lipoprotein that increase its atherogenicity. N Engl J Med 320:915-924

12. Diaz MN, Frei B, Vita JA, Keaney JF Jr (1997) Antioxidants and atherosclerotic heart disease. N Engl J Med 337:408-416

13. Mattioli AV, Palmiero P, Manfrini O, Puddu PE, Nodari S, Dei Cas A, Mercuro G, Scrutinio D, Palermo P, Sciomer S, Di Francesco S, Novo G, Novo S, Pedretti RFE, Zito A, Parati G, Pedrinelli R, Farinetti A, Maiello M, Moscucci F, Tenaglia RL, Sucato V, Triggiani M, Cugusi L, Scicchitano P, Saba PS, Ciccone MM (2017) Mediterranean diet impact on cardiovascular diseases: a narrative review. J Cardiovasc Med (Hagerstown) 18:925-935

14. Huang J, Weinstein SJ, Yu K, Männistö S, Albanes D (2018) Serum beta carotene and overall and cause-specific mortality. Circ Res 123:1339-1349

15. Goyal A, Terry MB, Siegel AB (2013) Serum antioxidant nutrients, vitamin A, and mortality in US Adults. Cancer EpidemiolBiomarkPrev 22:2202-2211

16. Hercberg S, Galan P, Preziosi P, Bertrais S, Mennen L, Malvy D, Roussel AM, Favier A, Briançon S (2004) TheSU.VI.MAX Study: a randomized, placebo-controlled trial of the health effects of antioxidant vitamins and minerals. Arch Intern Med 164:2335-2342

17. Blot WJ, Li JY, Taylor PR, Guo W, Dawsey S, Wang GQ, Yang CS, Zheng SF, Gail M, Li GY, Yu Y, Liu BQ, Tangrea J, Sun YH, Liu F, Fraumeni JF, Zhang YH, Li B (1993) Nutrition intervention trials in Linxian, China: supplementation with specific vitamin/ mineral combinations, cancer incidence, and disease-specific mortality in the general population. J Natl Cancer Inst 85:1483-1492

18. Tunstall-Pedoe H, Kuulasmaa K, Mahonen M, Tolonen H, Ruokokoski E, Amouyel P (1999) Contribution of trends in survival and coronary-event rates to changes in coronary heart disease mortality: 10-year results from 37 WHO MONICA project populations. Monitoring trends and determinants in cardiovascular disease. Lancet 353:1547-1557

19. Yarnell JW (1998) The PRIME study: classical risk factors do not explain the several fold differences in risk of coronary heart disease between France and Northern Ireland. Prospective epidemiological study of myocardial infarction. QJM 91:667-676

20. Yarnell J, Yu S, McCrum E, Arveiler D, Hass B, Dallongeville $\mathrm{J}$, Montaye M, Amouyel P, Ferrières J, Ruidavets JB, Evans A, 
Bingham A, Ducimetière P, PRIME study group (2005) Education, socioeconomic and lifestyle factors, and risk of coronary heart disease: the PRIME study. Int J Epidemiol 34:268-275

21. Yarnell JW, Patterson CC, Arveiler D, Amouyel P, Ferrières J, Woodside JV, Haas B, Montaye M, Ruidavets JB, Kee F, Evans A, Bingham A, Ducimetière P (2012) Contribution of lifetime smoking habit in France and Northern Ireland to country and socioeconomic differentials in mortality and cardiovascular incidence: the PRIME study. J Epidemiol Community Health 66:599-604

22. Wagner A, Simon C, Evans A, Ducimetière $P$, Bongard V, Montaye M, Arveiler D, PRIME Study Group (2003) Physical activity patterns in 50-59 year men in France and Northern Ireland. Associations with socio-economic status and health behaviour. Eur J Epidemiol 18:321-329

23. Ruidavets JB, Ducimetière P, Evans A, Montaye M, Haas B, Bingham A, Yarnell J, Amouyel P, Arveiler D, Kee F, Bongard V, Ferrières $\mathbf{J}$ (2010) Patterns of alcohol consumption and ischaemic heart disease in culturally divergent countries: the prospective epidemiological study of myocardial infarction (PRIME). BMJ 341:c6077

24. Dauchet L, Ferrières J, Arveiler D, Yarnell JW, Gey F, Ducimetière P, Ruidavets JB, Haas B, Evans A, Bingham A, Amouyel P, Dallongeville J (2004) Frequency of fruit and vegetable consumption and coronary heart disease in France and Northern Ireland: the PRIME study. Br J Nutr 92:963-972

25. Ducimetière $\mathrm{P}$, Ruidavets JB, Montaye M, Haas B, Yarnell J, PRIME Study Group (2001) Five-year incidence of angina pectoris and other forms of coronary heart disease in healthy men aged 50-59 in France and Northern Ireland: the prospective epidemiological study of myocardial infarction (PRIME) study. Int J Epidemiol 30:1057-1062

26. Hess D, Keller HE, Oberlin B, Bonfanti R, Schuep W (1991) Simultaneous determination of retinol, tocopherols, carotenes and lycopene in plasma by means of high-performance liquid chromatography on reversed phase. Int J Vitam Nutr Res 61:232-238

27. Vuilleumier JP, Keck E (1989) Fluorimetric assay of vitamin C in biological materials using a centrifugal analyser with fluorescence attachment. J Micronutr Anal 5:25-35

28. Gey KF, Brubacher GB, Stähelin HB (1987) Plasma levels of antioxidant vitamins in relation to ischemic heart disease and cancer. Am J ClinNutr 45:1368-1377

29. Gey KF, Moser UK, Jordan P, Stähelin HB, Eichholzer M, Lüdin E (1993) Increased risk of cardiovascular disease at suboptimal plasma concentrations of essential antioxidants: an epidemiological update with special attention to carotene and vitamin C. Am J ClinNutr 57(5 Suppl):787S-797S

30. Mayland CR, Bennett MI, Allan K (2005) Vitamin C deficiency in cancer patients. Palliat Med 19:17-20

31. Hickenbottom SJ, Follett JR, Lin Y, Dueker SR, Burri BJ, Neidlinger TR, Clifford AJ (2002) Variability in conversion of betacarotene to vitamin $\mathrm{A}$ in men as measured by using a doubletracer study design. Am J ClinNutr 75:900-907

32. Chai W, Maskarinec G, Franke AA, Monroe KR, Park SY, Kolonel LN, Wilkens LR, Le Marchand L, Cooney RV (2020) Association of serum $\gamma$-tocopherol levels with mortality: the multiethnic cohort study. Eur J ClinNutr 74:87-96

33. Parohan M, Anjom-Shoae J, Nasiri M, Khodadost M, Khatibi SR, Sadeghi O (2019) Dietary total antioxidant capacity and mortality from all causes, cardiovascular disease and cancer: a systematic review and dose-response meta-analysis of prospective cohort studies. Eur J Nutr 58:2175-2189

34. Bastide N, Dartois L, Dyevre V, Dossus L, Fagherazzi G, Serafini M, Boutron-Ruault MC (2017) Dietary antioxidant capacity and all-cause and cause-specific mortality in the E3N/EPIC cohort study. Eur J Nutr 56:1233-1243
35. Peng C, Luo WP, Zhang CX (2017) Fruit and vegetable intake and breast cancer prognosis: a meta-analysis of prospective cohort studies. Br J Nutr 117:737-749

36. Wang X, Ouyang Y, Liu J, Zhu M, Zhao G, Bao W, Hu FB (2014) Fruit and vegetable consumption and mortality from all causes, cardiovascular disease, and cancer: systematic review and dose-response meta-analysis of prospective cohort studies. BMJ 349: 84490

37. Browne D, Williams MA, Maxwell AP, McGuinness B, Passmore P, Silvestri G, Woodside JV, McKay GJ (2019) Serum xanthophyll carotenoids are associated with estimated glomerular filtration rate in an aged cohort. Sci Rep 9:17068

38. Thies F, Mills LM, Moir S, Masson LF (2017) Cardiovascular benefits of lycopene: fantasy or reality? ProcNutrSoc 76:122-129

39. Jialal I, Norkus EP, Cristol L, Grundy SM (1991) Beta-Carotene inhibits the oxidative modification of low-density lipoprotein. Biochim Biophys Acta 1086:134-138

40. Alpha-Tocopherol, Beta Carotene Cancer Prevention Study Group (1994) The effect of vitamin $E$ and beta carotene on the incidence of lung cancer and other cancers in male smokers. N Engl J Med 330:1029-1035

41. Browne D, McGuinness B, Woodside JV, McKay GJ (2019) Vitamin E and Alzheimer's Disease: what do we know so far? ClinInterv Aging 14:1303-1317

42. Bates CJ, Hamer M, Mishra GD (2011) Redox-modulatory vitamins and minerals that prospectively predict mortality in older British people: the National Diet and Nutrition Survey of people aged 65 years and over. Br J Nutr 105:123-132 ((Erratum in: Br J Nutr 2013;110:1548))

43. Martín-Calvo N, Martínez-González MÁ (2017) Vitamin C intake is inversely associated with cardiovascular mortality in a Cohort of Spanish Graduates: the SUN Project. Nutrients 9:E954

44. Grosso G, Bei R, Mistretta A, Marventano S, Calabrese G, Masuelli L, Giganti MG, Modesti A, Galvano F, Gazzolo D (2013) Effects of vitamin $\mathrm{C}$ on health: a review of evidence. Front Biosci (Landmark Ed) 18:1017-1029

45. Toniolo P, Van Kappel AL, Akhmedkhanov A, Ferrari P, Kato I, Shore RE, Riboli E (2001) Serum carotenoids and breast cancer. Am J Epidemiol 153:1142-1147

46. Mullan K, Cardwell CR, McGuinness B, Woodside JV, McKay GJ (2018) Plasma antioxidant status in patients with Alzheimer's Disease and cognitively intact elderly: a meta-analysis of casecontrol studies. J Alzheimers Dis 62:305-317

47. Mullan K, Williams MA, Cardwell CR, McGuinness B, Passmore P, Silvestri G, Woodside JV, McKay GJ (2017) Serum concentrations of vitamin E and carotenoids are altered in Alzheimer's disease: a case control study. Alzheimers Dement 3:432-439

48. Jenab M, Slimani N, Bictash M, Ferrari P, Bingham SA (2009) Biomarkers in nutritional epidemiology: applications, needs and new horizons. Hum Genet 125:507-525

49. Zheng JS, Sharp SJ, Imamura F, Chowdhury R, Gundersen TE, Steur M, Sluijs I, van der Schouw YT, Agudo A, Aune D, Barricarte A, Boeing H, Chirlaque MD, Dorronsoro M, Freisling H, ElFatouhi D, Franks PW, Fagherazzi G, Grioni S, Gunter MJ, Kyrø C, Katzke V, Kühn T, Khaw KT, Laouali N, Masala G, Nilsson PM, Overvad K, Panico S, Papier K, Quirós JR, Rolandsson O, Redondo-Sánchez D, Ricceri F, Schulze MB, Spijkerman AMW, Tjønneland A, Tong TYN, Tumino R, Weiderpass E, Danesh J, Butterworth AS, Riboli E, Forouhi NG, Wareham NJ (2020) Association of plasma biomarkers of fruit and vegetable intake with incident type 2 diabetes: EPIC-InterAct case-cohort study in eight European countries. BMJ 370:m219 